University of Wollongong

Research Online

Faculty of Engineering and Information

Faculty of Engineering and Information

Sciences - Papers: Part A

Sciences

$1-1-2014$

Potential of fluid pressure use for achieving high formability in micro sheet forming process

Hideki Sato

Tokyo Metropolitan University

Kenichi Manabe

Tokyo Metropolitan University

Dongbin Wei

University of Technology, Sydney, dwei@uow.edu.au

Zhengyi Jiang

University of Wollongong, jiang@uow.edu.au

Daiki Kondo

Tokyo Metropolitan University

Follow this and additional works at: https://ro.uow.edu.au/eispapers

Part of the Engineering Commons, and the Science and Technology Studies Commons

Research Online is the open access institutional repository for the University of Wollongong. For further information contact the UOW Library: research-pubs@uow.edu.au 


\title{
Potential of fluid pressure use for achieving high formability in micro sheet forming process
}

\author{
Abstract \\ This study decsribesa potential offluid pressure in micro hydromechanical deep drawingto achieve high \\ formability. The FE simulation is carried out using the stainless steel foil with $50 \mu \mathrm{m}$ thickness. The \\ friction holding effect can be obtained by applying the fluid pressure and it becomes high at high $D p / t$ in \\ MHDD. By applying the counter and radial pressure in MHDD, the desired lubrication condition can be \\ obtained and the friction force can be reduced. By the friction holding effect and lubrication effect in \\ MHDD, the micro cups can be successful fabricated in MHDD. Consequently, it is clarify that the fluid \\ pressure has a potential to achieve the high formability because it makes the friction holding, radial \\ pressure and lubrication effects in MHDD.
}

\section{Keywords}

potential, sheet, fluid, forming, process, pressure, achieving, high, formability, micro

Disciplines

Engineering | Science and Technology Studies

\section{Publication Details}

H. Sato, K. Manabe, D. Wei, Z. Jiang \& D. Kondo, "Potential of fluid pressure use for achieving high formability in micro sheet forming process," in International Conference of Computational Methods in Sciences and Engineering (ICCMSE), 2014, p. 327. 


\title{
Potential of Fluid Pressure Use for Achieving High Formability in Micro Sheet Forming Process
}

\author{
Hideki Sato $^{\mathrm{a}}$, Ken-ichi Manabe ${ }^{\mathrm{a}}$, Dongbin Wei ${ }^{\mathrm{b}}$, Zhengyi Jiang ${ }^{\mathrm{c}}$ and Daiki Kondo ${ }^{\mathrm{a}}$ \\ ${ }^{a}$ Department of Mechanical Engineering, Tokyo Metropolitan University, 1-1 Minamiosawa, Hachioji, Tokyo, \\ 192-0397, Japan \\ ${ }^{b}$ School of Electrical, Mechanical and Mechatronic Systems, University of Technology, Sydney, PO Box 123, \\ Broadway, NSW, 2007, Australia \\ ${ }^{c}$ School of Mechanical Materials and Mechatronic Engineering, University of Wollongong, Northfields Avenue, \\ Wollongong, NSW, 2522, Australia
}

\begin{abstract}
This study decsribes a potential of fluid pressure in micro hydromechanical deep drawing to achieve high formability. The FE simulation is carried out using the stainless steel foil with $50 \mu \mathrm{m}$ thickness. The friction holding effect can be obtained by applying the fluid pressure and it becomes high at high $D_{\mathrm{p}} / t$ in MHDD. By applying the counter and radial pressure in MHDD, the desired lubrication condition can be obtained and the friction force can be reduced. By the friction holding effect and lubrication effect in MHDD, the micro cups can be successful fabricated in MHDD. Consequently, it is clarify that the fluid pressure has a potential to achieve the high formability because it makes the friction holding, radial pressure and lubrication effects in MHDD.
\end{abstract}

Keywords: Micro sheet hydroforming, Micro hydromechanical deep drawing, Formability, Fluid pressure.

PACS: 62.20.fq, 62.20.Qp, 62.50.-p.

\section{INTRODUCTION}

Forming technology for achieving high formability is the key technology to produce the large quantities products, complex shape components and fabricate the hard-forming material. As the general forming technologies for sheet metal, the bending, blanking and deep drawing have been used. Due to parts and products miniaturizations, the scaling down of these forming technologies has become a research focus for the last several years [1]. However, with miniaturization of target size, the deformation behavior changes and the formability decreases due to the size effects. Fu et al. investigated the grain size effect on fracture behavior in tensile test [2]. They found that the fracture stress and strain decrease in micro scale due to the small ratio of thickness to grain size. Saotome et al. revealed that the relative punch diameter to thickness becomes small in micro scale and it causes the change of dominate deformation mode [3]. Moreover, Putten et al. reported the friction coefficient increases with scaling down [4]. Due to these size effects in micro scale, the formability in micro forming becomes low.

To improve the formability in micro scale, a micro hydromechanical deep drawing (MHDD) has been developed by Authors [5]. In this forming method, it is expected that the formability can be improved by the advantages of sheet hydroforming, such as hydrodynamic lubrication and friction holding effect. Previously, the effect of fluid pressure of deformation behavior and the tribological size effect in MHDD have been clarified [6-7]. This study introduces the potential of fluid pressure to achieve high formability in micro sheet forming.

\section{FE SIMULATION}

FE simulations of the conventional MDD and MHDD were carried out using the commercial FE software LSDYNA ver.971. The material used is the stainless steel foil (SUS304-H) with $50 \mu \mathrm{m}$ thickness. The mechanical properties are listed in Table 1. The real stress-strain data obtained by tensile test was employed in the material model. The tool dimensions of MDD and MHDD at different $D_{\mathrm{p}} / t$ are listed in Table 2 . The full model was modeled. The blank and tools were modeled by the isotopic elastoplastic of solid element and the rigid of shell element, respectively. The two kind of fluid pressures, counter and radial pressures were $p=10,30,60$ and $100 \mathrm{MPa}$ in MHDD and applied on the blank surface and from the blank edge, respectively. 
TABLE 1. Mechanical properties used at FE simulation.

\begin{tabular}{cccc}
\hline $\begin{array}{c}\text { Yield stress, } \\
\sigma_{\mathrm{y}}[\mathrm{MPa}]\end{array}$ & $\begin{array}{c}\text { Young's modulus, } \\
E[\mathrm{GPa}]\end{array}$ & $\begin{array}{c}\text { Tensile strength, } \\
\sigma_{\mathrm{B}}[\mathrm{MPa}]\end{array}$ & $\begin{array}{c}\text { Elongation, } \\
\delta[\%]\end{array}$ \\
\hline 1217 & 192 & 1334 & 2.4 \\
\hline
\end{tabular}

TABLE 2. Tool dimension of MDD and MHDD at different $D_{\mathrm{p}} / t$.

\begin{tabular}{cccc}
\hline$D_{\mathrm{p}} / t$ & 20 & 38 & 100 \\
\hline Blanking punch diameter, $D_{\mathrm{o}}[\mathrm{mm}]$ & 1.737 & 3.300 & 8.684 \\
Drawing punch diameter, $D_{\mathrm{p}}[\mathrm{mm}]$ & 1.0 & 1.9 & 5.0 \\
Drawing punch shoulder radius, $r_{\mathrm{p}}[\mathrm{mm}]$ & 0.053 & 0.100 & 0.263 \\
Drawing die diameter, $D_{\mathrm{d}}[\mathrm{mm}]$ & 1.140 & 2.040 & 5.140 \\
Drawing die shoulder radius, $r_{\mathrm{d}}[\mathrm{mm}]$ & 0.105 & 0.200 & 0.526 \\
Blank holder constant gap, $h[\mathrm{~mm}]$ & 0.06 & 0.06 & 0.06 \\
\hline
\end{tabular}

\section{RESULTS AND DESCUSSION}

\section{Friction holding effect in MHDD}

The friction holing force is known as the friction force between the punch and blank at side wall to reduce the loading force of blank at punch shoulder. This friction holding effect can be evaluated by the straightness at side wall. When the blank contacts the punch at side wall, the friction force occurs between the blank and punch, which is called the friction holding force. At the same time, the straightness at side wall becomes zero due to the contact between the blank and punch. Therefore, the occurrence of friction holding effect can be evaluated by the straightness of side wall. Figure 1 shows the effect of fluid pressure on normalized straightness at conventional MDD and MHDD. At $D_{\mathrm{p}} / t=38$, the straightness is 0 at MHDD with fluid pressure of $60 \mathrm{MPa}$ although it is 0.006 at MDD. The straightness increases with the increase of fluid pressure. It is because the fluid pressure pushes the side wall of blank during the forming and the blank fits with the punch. On the other hand, the $D_{\mathrm{p}} / t$ also affects the straightness at side wall. At small $D_{\mathrm{p}} / t$, the foil thickness is relatively thick to the punch diameter and the bending resistance becomes high at punch shoulder part. Therefore the high fluid pressure is required to make a contact between the blank and punch at side wall. In contrast, at high $D_{\mathrm{p}} / t$, the bending resistance decreases because the foil thickness is relatively thin. It makes easy to obtain the contact between the blank and die at high $D_{\mathrm{p}} / t$. It was found that the friction holding effect can be obtained in MHDD with high $D_{\mathrm{p}} / t$.

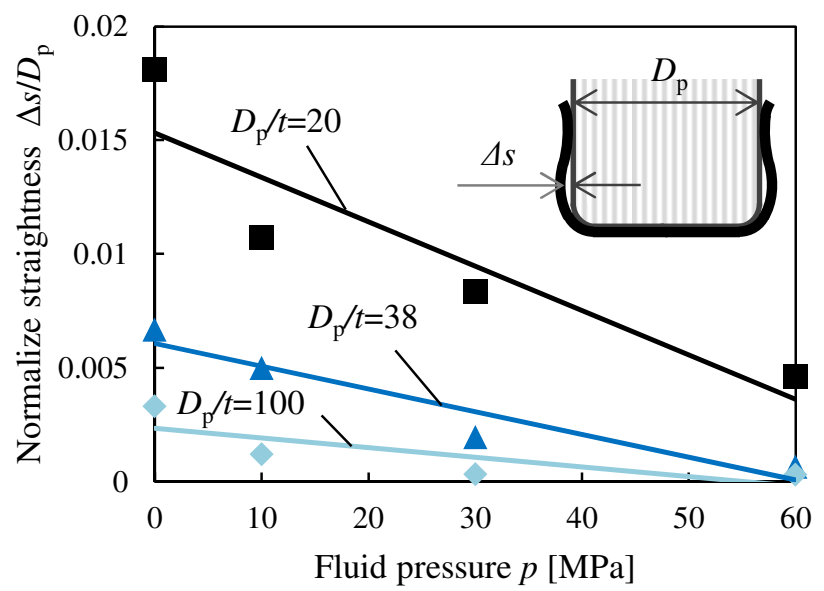

FIGURE 1. Effect of fluid pressure on straightness at different relative punch diameters $D_{\mathrm{p}} / t$. 


\section{Lubrication by counter and radial pressures in MHDD}

The lubrication conditions in MDD and MHDD are divided into seven types as shown in Figure 2(a). In conventional MDD, the lubricant cannot be kept at the outer pockets area of blank because the outer pockets connect with the blank edge. In addition, the ratio of outer pockets to blank surface increases with scaling down. Therefore, the friction force becomes high in MDD even though the lubricant is applied [4]. In contrast, the lubricant can be kept at outer pockets area by applying the fluid pressure in MHDD as shown in Fig. 2(a). It was reported that the friction force in the lubrication at outer pockets (lubrication condition 4) becomes small as compared with the dry friction and lubrication conditions in MDD [7]. By making the hydrodynamic lubrication (lubrication conditions 5 and 6), the friction coefficient becomes around 0.005 and the friction force significantly decreases. In addition, in the both hydrodynamic lubrication (lubrication condition 7), the friction force becomes quite small. It has been revealed that the friction coefficient becomes small by making the lubrication conditions 4, 5, 6 or 7 .

These lubrication conditions in MHDD can be made by applying two kinds of fluid pressures, counter pressure $p_{\mathrm{cp}}$ and the radial pressure $p_{\mathrm{rp}}$ which are applied from the die cavity and blank edge, respectively as shown in Fig. 2(a). The counter and radial pressures play different roles for the lubrication. For the counter pressure, there is no leakage when $p_{\mathrm{cp}}$ is under $q_{\mathrm{d}}$ because the generated counter pressure is not enough to leak between the die and blank. When $p_{\mathrm{cp}}$ exceeds $q_{\mathrm{d}}$, the fluid medium leaks and the hydrodynamic lubrication can be obtained between the die and blank. At the same time, the fluid medium inserts the outer pockets area and this area is lubricated [7]. When $p_{\mathrm{cp}}$ exceeds the total amount of contact pressures between the blank and blank holder $q_{\mathrm{b}}$ and $q_{\mathrm{d}}$, the leakage can be resulted not only between the blank and die, but also between the blank holder and blank. Therefore, the counter pressure can make the leakage between the blank and die first and then the leakage between the blank and blank holder. On the other hand, for the radial pressure, the fluid medium can insert the outer pockets directly without the leakage. Moreover, the hydrodynamic lubrication between the blank, blank holder and die can be obtained when $p_{\text {rp }}$ exceeds $q_{\mathrm{b}}$ and $q_{\mathrm{d}}$, respectively. It means that the both hydrodynamic lubrication can be obtained when $p_{\text {rp }}$ exceeds $q_{\mathrm{d}}$. Therefore, the required radial pressure for the both hydrodynamic lubrication is smaller than the counter pressure. Finally, the area of counter and radial pressures to obtain each lubrication condition in MDD and MHDD can be expressed as Fig. 2 (b). From these results, it can be said that there is a potential to obtain the small friction force in the area 4, 5, 6 and 7 .

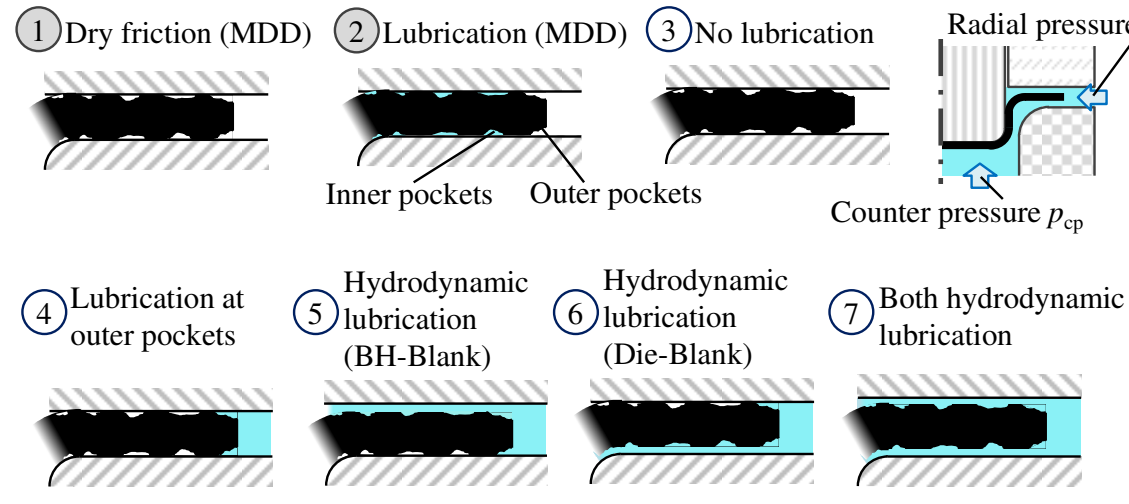

(a) Lubrication condition in MDD and MHDD

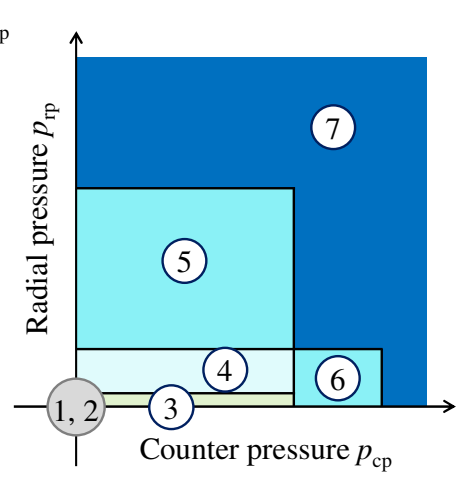

(b) Required counter and radial pressures for obtaining each lubrication condition

FIGURE 2. Classification of lubrication conditions and required counter and radial pressures in MDD and MHDD

\section{Formability in conventional micro sheet forming and micro sheet hydroforming}

Figure 3 shows the fracture risk at conventional micro sheet forming and micro sheet hydroforming. The maximum effective punch force is normalized by the tensile strength. When this value exceeds 1 , the punch force exceeds the tensile strength and the fracture is resulted. Therefore the fracture risk can be evaluated by the normalized maximum effective punch force. At conventional micro sheet forming, the fracture risk increases with increases of $D_{\mathrm{p}} / t$. In particular, the fracture risk reaches 0.9 and is quite high at $D_{\mathrm{p}} / t=100$. It is because the normalized friction force increases as $D_{\mathrm{p}} / t$ increases. The same result is confirmed experimentally by Saotome et al. [3]. Thus, the low formability in conventional micro sheet forming is mainly resulted by the high friction force. Therefore, the high formability area is limited only at small $D_{\mathrm{p}} / t$. On the other hand, at micro sheet hydroforming, 
the friction coefficient can be reduced by applying the fluid pressure. Therefore, the fracture risk is smaller than that at conventional micro sheet forming due to the high lubrication by the fluid pressure. Also, by applying the fluid pressure, the friction holding effect can be obtained at the high $D_{\mathrm{p}} / t$. Moreover, the radial pressure compresses the blank edge and reduces the loading force of blank. As above, by the lubrication, friction holding and radial pressure effects in MHDD, the micro cup of high drawing ratio can be successfully drawn in MHDD although it cannot be fabricated in MDD as shown in Figure 4. Due to the effects of fluid pressure in MHDD, the cup height was improved over twice. Consequently, it was confirmed that the fluid pressure has a potential to achieve the high formability in MHDD by the lubrication, friction holding and radial pressure effects.

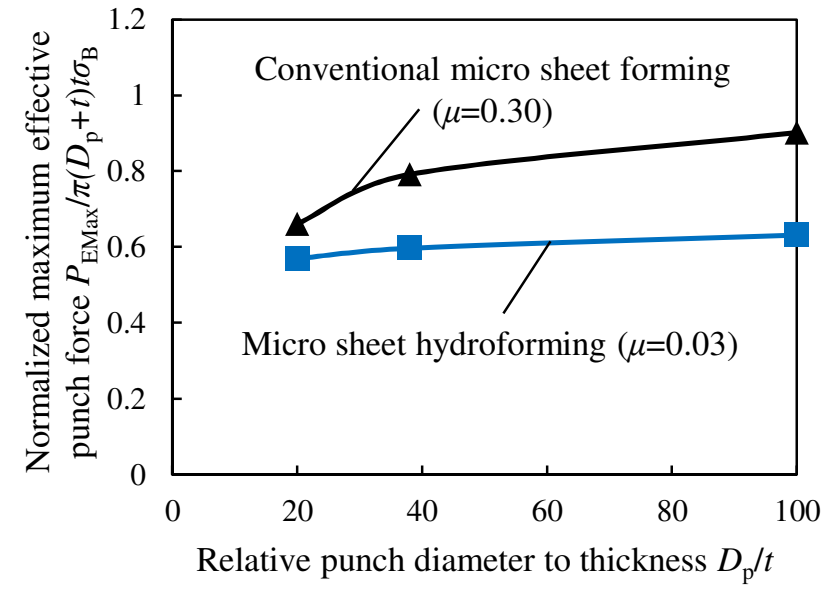

FIGURE 3. Fracture risk at conventional micro sheet forming and micro sheet hydroforming.

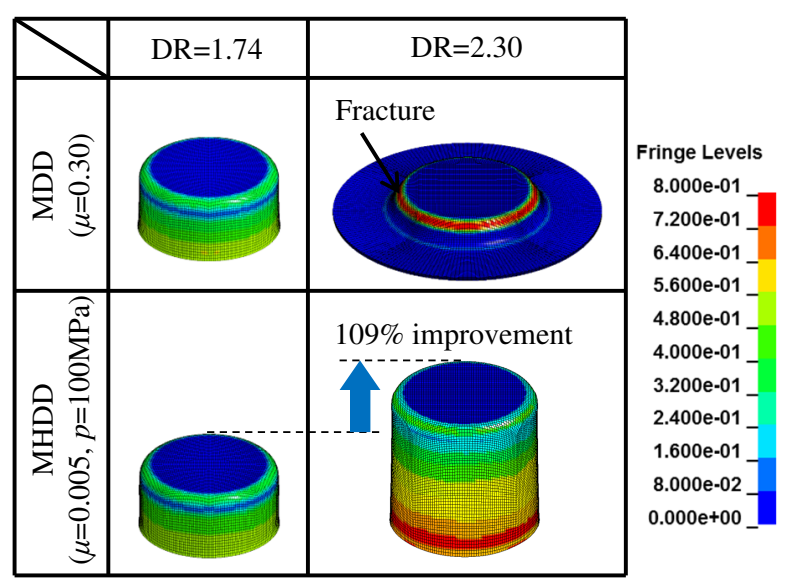

FIGURE 4. Appearance of cups and its plastic distribution at different drawing ratios.

\section{CONCLUSION}

In this study, the potential of fluid pressure to achieve high formability in micro sheet forming is investigated. By applying the fluid pressure in MHDD, the friction holding effect can be obtained. Its effect becomes high especially at high $D_{\mathrm{p}} / t$. By controlling the amount of counter and radial pressures, the desired lubrication condition can be obtained in MHDD and the friction force can be significantly reduced as compared with conventional MDD. Finally, it was found that the fluid pressure has a potential to achieve high formability at MHDD due to the effect of fluid pressure which can obtain the friction holding, radial pressure and lubrication effects.

\section{REFERENCES}

1. M. Geiger, M. Kleiner, R. Eckstein, N. Tiesler, U. Engel, Microforming, CIRP Annals - Manufacturing highlights, 50, (2001), pp. 445-462.

2. M. W. Fu, W. L. Chan, Geometry and Grain Size Effects on the Fracture Behavior of Sheet Metal in Micro-Scale Plastic Deformation, Materials and Design, Vol.32, (2011), pp.4738-4746.

3. Y. Saotome, K.. Yasuda, H. Kaga, Microdeep Drawability of Very Thin Sheet Steels, Journal of Materials Processing Technology, Vol.113, (2001), pp.641-647.

4. K.V. Putten, M. Franzke, G. Hirt, Size Effect on Friction and Yielding in Wire Flat Rolling, Proceedings of the 2nd International Conference on New Forming Technology, (2007), pp.583-592.

5. H. Sato, K. Manabe, K. Ito, D. Wei, Z. Jiang, Development of Servo-Type Micro-Hydromechanical Deep-Drawing Apparatus and Micro Deep-Drawing Experiments of Circular Cups, Journal of the Japan Society for Technology of Plasticity (in Japanese), Vol.55, (2014), pp.44-49.

6. H. Sato, K, Manabe, D. Wei, Z. Jiang, Effect of Fluid Pressure in Micro Hydromechanical Deep Drawing, Proceedings of the $6^{\text {th }}$ International Conference on Tube Hydroforming, (2013), pp. 313-317.

7. H. Sato, K, Manabe, D. Wei, Z. Jiang, Numerical Modeling of Size Effect in Micro Hydromechanical Deep Drawing, Proceedings of the $9^{\text {th }}$ International Conference and Workshop on Numerical Simulation of 3D Sheet Metal Forming Processes, (2013), In press. 